\title{
Thermal Separation: An Approach for a Modelica Library for Absorption, Adsorption and Rectification
}

\author{
Andreas Joos* Karin Dietl $^{\dagger}$ Gerhard Schmitz ${ }^{\ddagger}$ \\ Hamburg University of Technology \\ Institute of Thermo-Fluid Dynamics ${ }^{\S}$ Applied Thermodynamics \\ 21071 Hamburg, Germany
}

\begin{abstract}
Due to its objected-oriented design Modelica is predestinated to describe chemical engineering unit operations. Still only few activity on this field is published. This paper introduces a library which covers three of four major thermal seperation processes: absorption, adsorption and rectification.

Additionally an extension of the ExternalMedia library is presented that allows the connection to thermodynamic and physical property packages for two phase mixtures. Also a possible forecast to an implementation of a CAPE-OPEN interface is made, allowing the use of a plurality of established chemical data packages.

Keywords: Thermal Separation, Separation Column, ExternalMedia, Heat and Mass Transfer
\end{abstract}

\section{Introduction}

Global warming has become a major issue in society and politics. The reduction of $\mathrm{CO}_{2}$-emission of power plants plays an important role when talking about reduction of $\mathrm{CO}_{2}$-emission. One possibility is to remove the $\mathrm{CO}_{2}$ from the exhaust gas via absorption, liquefy it and store it underground. In order to optimize this process dynamic modeling is very important since for example the composition of the exhaust gases vary during operation. Whereas there exist already libraries for the dynamic modeling of power plants (like for instance the free Modelica library ThermoPower [2]), Modelica models to model the $\mathrm{CO}_{2}$ - separation process have not been found in literature.

However not only $\mathrm{CO}_{2}$ - separation is an interesting topic of dynamic modeling but also other separation

\footnotetext{
*email: andreas.joos@tu-harburg.de, Tel.:+49 40428783079

†email: karin.dietl@ tu-harburg.de

†email: schmitz@tu-harburg.de, Tel.:+49 40428783144

$\S_{\text {http: / / www.tu-harburg.de/tt }}$
}

processes like adsorption or rectification. A dynamic analysis of such processes gains in importance as dynamic process strategies becoming more popular, be it in batch processing or start-up strategies in continuous processing. Since ad- and absorption as well as rectification have much in common from a modeling point of view, the development of a combined separation library is proposed. The fourth common separation process, extraction, differs from the other processes and is therefore not considered up to now.

\section{Modeling}

\subsection{Library Structure}

The library structure can be seen in figure 1 which shows a class diagram of the library. There exist three different models: one of the packed column, one of the tray column and one of the spray column. They all extend from BaseStageVL. This base class contains the mole and energy balances for $n$ stages, an instance of the PhaseBoundary-model and of the medium models as well as the instances of the connector classes. The extending classes supply the geometry, the instances of the pressure loss model, the heat transfer model between the two phases and the mass transfer model. Each column type is structured the same; but only the structure of the packed column is shown in the diagram. For each column type a different class exists. They differ in the models which they allow to replace their base classes. This prevents the user to choose for example in a tray column a pressure loss model developed for a packed column.

\subsection{Interfaces}

Columns can be connected to other columns or to fluid sources or sinks using connectors. The connector for the vapour flow takes the volume flow rate as flow vari- 


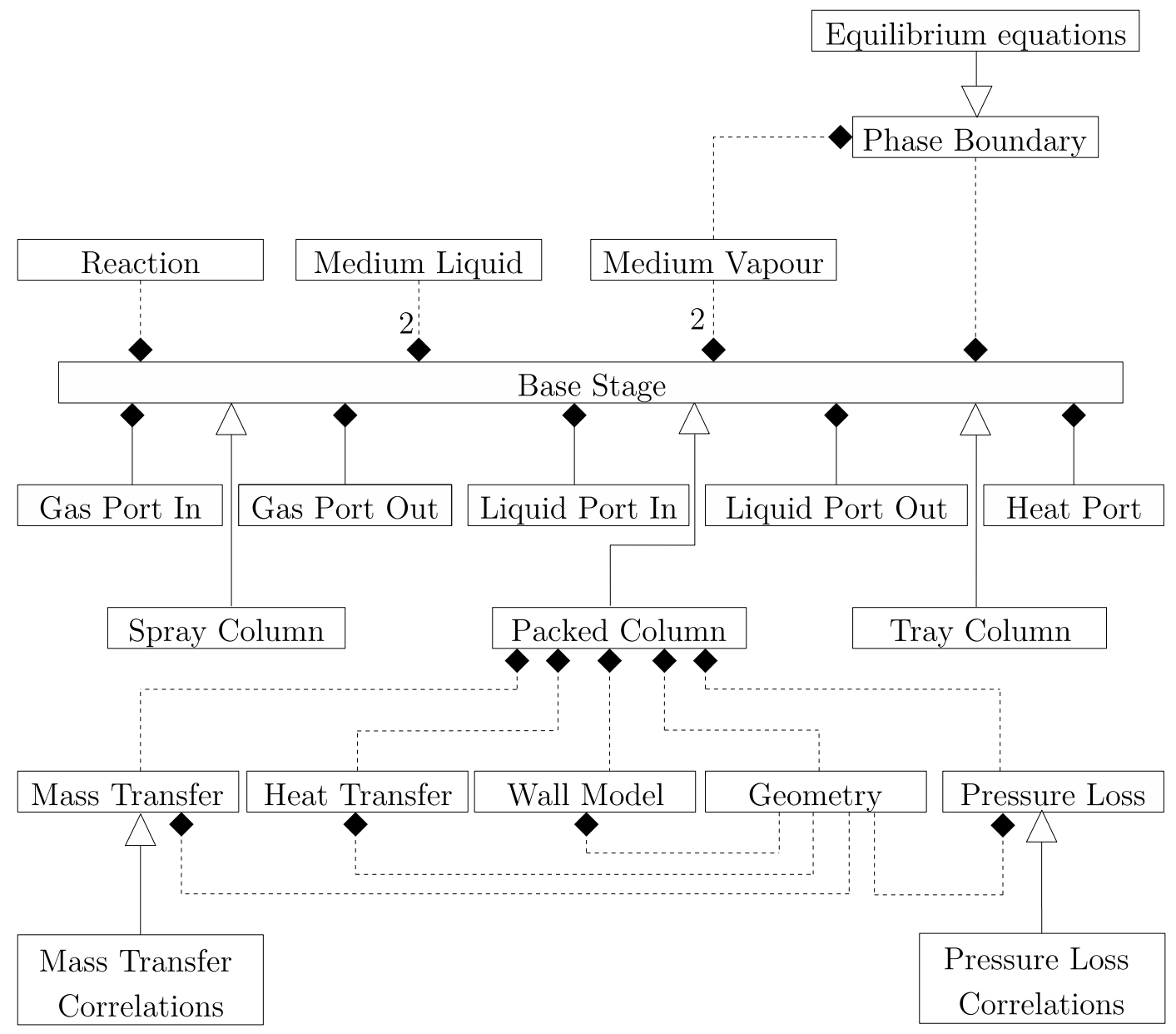

Figure 1: UML class diagram of an absorption or rectification process. The arrow denotes inheritance, the line with the diamond denotes composition. Dotted lines mean that the object is replaceable. The composition of spray column and tray column are analogue to the composition of the packed column. However, this is not shown in the class diagram due to readability.

able and the pressure as potential variable and temperature, molar concentration and composition as in/outputs. The connectors for the liquid flow are the same, only the pressure as potential variable is replaced by the height. Each column also contains a heat port to account for heat losses to ambiance.

\subsection{Assumptions}

The following assumptions hold for the modeling:

- discretization only in axial direction

- counterflow of vapour and liquid flow

- no entrainment of the liquid with the vapour

- tray columns: no raining through the plates

- reaction, if any, takes place in the liquid phase
- spray columns: drops move only in axial direction

- spray columns: no coalescence or splitting of drops

- no accumulation in gas and liquid films

- no second liquid phase possible

\subsection{Modeling of one discrete element}

One major design criterion of the library is to take into account heat and mass transfer between the phases; i.e. consideration of non-equilibrium states; equilibrium is only attained at the phase boundary. This is illustrated in figure 2: Therefore the balance equations for heat as well as for mass have to be written in each discrete element for every phase, so a molar flux tends to eliminate the difference between actual composition $x_{A}$ and 
equilibrium composition $x_{A}^{*}$ and a heat flux tends to equal the vapor temperature $T_{v}$ and the temperature of the liquid phase $T_{l}$. Even though in general the temperature of vapour and liquid phase are almost the same, the introduction of a heat flow rate $\dot{Q}_{t}$ between the two phases makes sense, since it simplifies the calculation.

The Basestage-model contains $n$ discrete elements for which mole and energy balances are established.

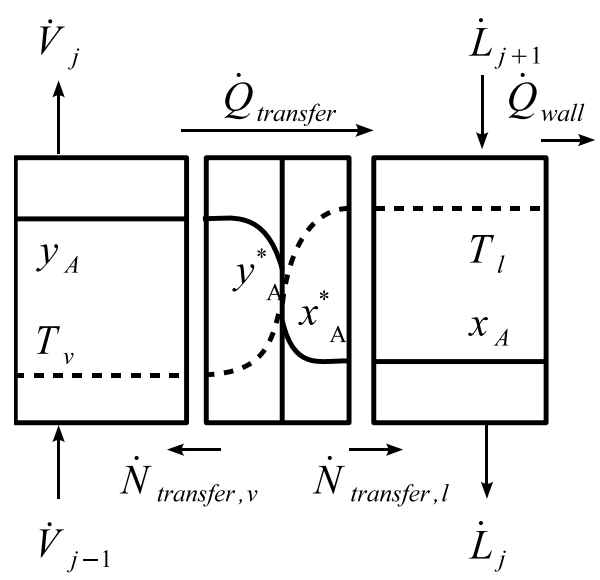

Figure 2: Sketch of the control volumes and the phase boundary

The mole balance for the stage $j$ and the component $i$ of the vapour phase and the liquid phase respectively are as follows:

$$
\begin{gathered}
V \cdot \varepsilon \cdot \frac{d}{d t}\left(\varepsilon_{v, j} \cdot c_{v, i, j}\right)= \\
\dot{V}_{j-1} \cdot c_{v, i, j-1}-\dot{V}_{j} \cdot c_{v, i, j}+\dot{N}_{v, t, i, j} \\
V \cdot \varepsilon \cdot \frac{d}{d t}\left(\varepsilon_{l, j} \cdot c_{l, i, j}\right)= \\
\dot{L}_{j+1} \cdot c_{l, i, j+1}-\dot{L}_{j} \cdot c_{l, i, j}+\dot{N}_{l, t, i, j}+\dot{N}_{\text {reaction }, i, j}
\end{gathered}
$$

Since no mass is accumulated in the gas or liquid film, the molar fluxes sum up to zero:

$$
\dot{N}_{v, t, i, j}+\dot{N}_{l, t, i, j}=0
$$

The vectors $\dot{N}_{v, t, j}$ and $\dot{N}_{l, t, j}$ contain the molar flux of each component. If the component is entering the control volume, the sign is positive, otherwise negative.

The energy balance is also established for the two phases separately. Solid material in the column (trays and packing material) are supposed to have the same temperature as the liquid phase. Heat transfer to ambiance also takes place via the liquid phase. Thus for the element $j$ the energy balance for the liquid phase becomes:

$$
\begin{array}{r}
V \cdot \varepsilon \cdot \frac{d}{d t}\left(\varepsilon_{l, j} \cdot u_{l, j}\right)+V \cdot \rho_{s} \cdot c_{s} \cdot \frac{d T_{l}}{d t}= \\
\dot{L}_{j+1} \cdot h_{l, j+1}-\dot{L}_{j} \cdot h_{l, j}-\dot{Q}_{\text {wall }, j} \\
+\dot{Q}_{t}+\dot{H}_{\text {fromV }, j}-\dot{H}_{\text {fromL }, j}
\end{array}
$$

and for the vapour phase:

$$
\begin{array}{r}
V \cdot \varepsilon \cdot \frac{d}{d t}\left(\varepsilon_{v, j} \cdot u_{v, j}\right)=\dot{V}_{j-1} \cdot h_{v, j-1}-\dot{V}_{j} \cdot h_{v, j} \\
+\dot{Q}_{t}+\dot{H}_{\text {fromL }, j}-\dot{H}_{\text {fromV }, j}
\end{array}
$$

Using the medium models, the molar specific inner energies and enthalpies are calculated for a certain temperature, pressure and mole fraction. The volume specific inner energies and enthalpies are then calculated using the concentration, for example:

$$
u_{l, j}=\sum_{i=1}^{n S} c_{l, i, j} \cdot u_{l, j}^{m}
$$

Together with the molar flux over the phase boundary there is also an enthalpy flux over the phase boundary. Since obviously the composition of the condensing vapour stream differs from the composition of the vapour bulk phase a second instance of the vapour medium, called mediumVapourTransfer exists. In there the thermodynamic properties of the transfer vapour stream are calculated, using temperature and pressure of the bulk phase but the composition of the transfer stream. The liquid is treated accordingly. The specific enthalpy obtained by mediumVapourTransfer and mediumLiquidTransfer is used to calculate the two enthalpy flows, $\dot{H}_{\text {fromV }, j}$ and $\dot{H}_{\text {fromL }, j}$.

Additionally to the differential equations above, there exist also a differential equation for the liquid mass of each element:

$$
\begin{array}{r}
V \cdot \frac{d}{d t}\left(\varepsilon_{l, j} \cdot \rho_{l, j}\right)=\dot{L}_{j+1} \cdot \rho_{l, j+1}-\dot{L}_{j} \cdot \rho_{l, j} \\
+\sum_{i=1}^{n S} \dot{N}_{l, t, i, j} \cdot M_{l, j}
\end{array}
$$

\subsection{Mass Transfer and Phase Equilibrium}

The molar flow rate which transfers the phase boundary is calculated using the mass transfer resistance on the liquid and the vapour side and the equilibrium concentration at the phase boundary. However the determination of the mass transfer coefficient and of the mass transfer area (especially for tray columns) can be 
very difficult and the result very inaccurate. Therefore often equilibrium is assumed on every stage. The number $n$ of discrete elements for spray and packed column is then calculated as $n=H /$ HETP, where HETP is the height equivalent to one theoretical plate. For the tray columns, equilibrium is assumed on every tray. The deviation from equilibrium is then taken into account using the Murphree tray efficiency $\eta_{\text {Murphree }}$ :

$$
\eta_{\text {Murphree }, i, j}=\frac{y_{i, j}-y_{i, j-1}}{y_{i, j}^{*}-y_{i, j-1}}
$$

The thermodynamic equilibrium at the phase boundary is generally calculated using

$$
y_{j, i}^{*} \cdot p_{j} \cdot \phi_{v, j, i}=x_{j, i}^{*} \cdot \gamma_{j, i} \cdot \phi_{l, j, i} \cdot p_{j, i}^{s a t}
$$

This equation is valid in case the pressure is not extremely high, since the Poyinting-factor is neglected. For the activity coefficient $\gamma$ up to now Margules twosuffix equation, Wilson-equation and NRTL-equation are implemented. If the fugacity coefficient $\phi_{v}$ is not supposed to be 1, it can be calculated using the virial equation or a cubic equation of state after RedlichKwong (see for example [10]). In absorption processes the simpler Henry's law is often used. The equilibrium using Henry's law becomes:

$$
y_{j, i}^{*} \cdot p_{j} \cdot \phi_{v, j, i}=x_{j, i}^{*} \cdot \mathrm{He}_{j, i}
$$

The temperature dependency of the Henry-constant He is taken into account. The user can decide for each component whether equation (9) or (10) is used.

If mass transfer is taken into account the transfer molar flow rate is calculated using the difference between the concentration on the phase boundary and the bulk concentration. The molar vapour flow becomes:

$$
\dot{N}_{v, t, i, j}=-k_{v, i, j} \cdot A \cdot\left(c_{v, i, j}^{b u l k}-c_{v, i, j}^{*}\right)
$$

For the liquid molar flow rate additionally an enhancement factor is introduced:

$$
\dot{N}_{l, t, i, j}=-E_{i, j} \cdot k_{l, i, j} \cdot A \cdot\left(c_{l, i, j}^{b u l k}-c_{l, i, j}^{*}\right)
$$

The enhancement factor $E$ can be used if chemical reaction occurs. If for example the absorbed gas reacts with one or more liquid components, the mass transfer is enhanced due to the reaction. This influence is very important for fast reactions, where the reaction takes place in the liquid film. For slow reactions, which take place in the liquid bulk phase, the mass transport is only influenced indirectly and the enhancement factor becomes 1 . The determination of the enhancement factor can become very complex, especially if parallel reactions interact with each other. An overview can be found in [13]. In this library the equations for the following cases are implemented: irreversible first order reaction, pseudo-irreversible first order reaction, reversible reactions of type $\mathrm{A} \leftrightarrow \mathrm{P}, \mathrm{A} \leftrightarrow 2 \mathrm{P}$ and $\mathrm{A}+\mathrm{B} \leftrightarrow \mathrm{P}$. Also parallel, non-interacting reactions can be taken into account.

The liquid and vapour mass transfer coefficient for tray columns is calculated by correlations proposed by [12]. The interfacial area can be calculated for drop regime and emulsion regime; for the regime in between an interpolation, also proposed by [12], is used, using the ratio vapour load / maximum vapour load. For packed columns the interfacial area is simply the area of the packing material. The liquid and vapour mass transfer coefficients can either be calculated by the correlation from Onda or by using the Sherwoodnumber from Wesselingh (see for example [7]).

\subsection{Liquid and Vapour Flow Rate}

For different column types different equations for the liquid volume flow rate have to be used. The equations are directly implemented in the column model. Tray and packed column account for the case where at simulation start no liquid is in the column. In this case the outgoing liquid flow rate $\dot{L}$ is zero, unless the height of the liquid on the tray gets higher than the weir height (tray column) or the packing material is fully wetted (packed column). For a spray column it is assumed that if liquid enters the column, instantaneously liquid is also leaving. Hereby the liquid volume flow rate may change over the column height, but the number of drops per second remains the same. Therefore the liquid volume flow rate for the spray column is simply:

$$
\dot{L}_{j}=v_{\mathrm{drop}, j} \cdot A \cdot \varepsilon_{l}
$$

The velocity of one liquid drop can be obtained by a simple balance of forces on one liquid drop [8] and depends on vapour and liquid density, vapour viscosity, vapour velocity and the diameter of the liquid drop at the inlet. For the tray column the equation from [12] was used:

$$
\dot{L}_{j}= \begin{cases}l_{w} \cdot \varepsilon_{l, 2 p h, j} \cdot\left(\frac{\left(h_{j}-h_{w}\right) \cdot g^{1 / 3}}{1.45}\right)^{3 / 2} & \text { if } h>h_{w} \\ 0 & \text { else }\end{cases}
$$

$l_{w}$ denotes the weir length and $\varepsilon_{l, 2 p h}$ the liquid fraction in the two-phase regime on the tray. 
For packed columns the liquid volume flow rate is 0 , as long as the packing material is not yet wetted, otherwise

$$
\dot{L}_{j}=\sqrt{\left(\frac{\varepsilon_{l, j}}{0.555}\right)^{3} \cdot \frac{g \cdot \varepsilon^{4.65}}{a}} \cdot A \cdot \varepsilon \cdot \varepsilon_{l, j} \cdot 50
$$

As for the liquid flow rate, also for the determination of the vapour flow rate different equations for the different column types have to be used. In all cases, the momentum balances are formed across the segment boundaries (staggered grid), as was proposed by [3]. The used pressure drop correlations for the three column types are written below:

Tray column:

$$
p_{j}-p_{j+1}=\left(\frac{\dot{V}_{j}}{A_{\text {free }}}\right)^{2} \cdot \zeta \cdot \frac{\rho_{v, j}}{2}+h_{j} \cdot \varepsilon_{l, 2 p h, j} \cdot \rho_{v} \cdot g
$$

Spray column:

$$
p_{j}-p_{j+1}=\left(\frac{\dot{V}_{j}}{A_{\text {free }}}\right)^{2} \cdot \frac{\rho_{v, j}}{2} \cdot \lambda \cdot \frac{h_{j}}{d}
$$

Packed column:

$$
p_{j}-p_{j+1}=\frac{1}{8} \zeta\left(\frac{\dot{V}_{j}}{A_{\text {free }}}\right)^{2} \cdot\left(\frac{6 \varepsilon_{l}}{d_{L}}+a\right) \cdot \frac{\rho_{v, j} \cdot h_{j}}{\left(\varepsilon-\varepsilon_{l}\right)^{4.65}}
$$

Since the staggered grid approach is used, for the pressure difference $p_{i n}-p_{1}$ and for the pressure difference $p_{n}-p_{n+1}$ the height of the discrete element, $h$, is divided by 2 .

In order to make things easier for the nonlinear solver, the equations are written in the form $\dot{V}_{j}=\ldots$; only for the inlet volume flow rate the equation remains $p_{\text {in }}=f k t\left(\dot{V}_{\text {in }}, p_{1}, e t c.\right)$.

\section{Media Modeling}

\subsection{Physical and Thermodynamic Property Package}

A very important part when modeling separation processes are the medium models. As shown in figure 1, models for a liquid and a gas phase as well as a model for the equilibrium at the phase boundary are necessary.

The Modelica Standard Library provides the Modelica.Media package. This package provides medium models of ideal mixtures of ideal gases and liquids. If this phase behavior is not adequate for the modeled process other medium models are needed. Since implementing the mediums of interest in Modelica is very time consuming and complex, an external media interface to existing physical and thermodynamic property packages is preferable in such a case. Casella and Richter proposed the ExternalMedia library in order to include external fluid property code, [4]. As an example this library provides the interface to FluidProp of TU Delft [6] which is itself an interface to different medium databases.

The ExternalMedia library however only provides access to multi-phase pure substances but not for multi-phase-multi-component mediums. Since the authors of the ExternalMedia library provided access to the $\mathrm{C}++$ interface, this interface was changed in order to have access to the mixtures also.

Figure 3 shows a class diagram of the modified interface layer consisting of $\mathrm{C}++$ objects. Beside the TwoPhaseMedium a TwoPhaseMixture object was introduced to handle the extra functionality that is needed to compute the properties of a mixture in contrast to a pure substance. To make the new object to fit in the library structure and to preserve the present interface some of the existing classes had to be changed. Most of these changes were expansions of the former function templates to accept parameter for the mixtures like nComp for the number of components. The additional parameters were located at the end of the argument list of each functions and given default values. For example the class TwoPhaseMedium could remain unchanged, although arguments were added to functions in BaseTwoPhaseMedium allowing TwoPhaseMixture to inherit. To access the mixture properties via the TwoPhaseMixture object functions were added to the Modelica ExternalMediaLib front-end and the $\mathrm{C}$ interface layer.

Using two phase mixtures from the NIST Reference Fluid Thermodynamic and Transport Properties Database (REFPROP) [9] with the ExternalMedia library by the FluidProp interface shaped up to be quite inappropriate. First the calculation of properties from pressure, enthalpy and composition are quite slow, about $50 \mathrm{~ms}$ per function call. This leads due to several hundred to thousand function calls per time step to very large simulation times. Second some functions seem to be discontinuous, which can cause the simulation to crash. Both of these facts are disadvantageous above all in iterations of the non-linear solver.

However it would be a very useful tool not only for this library to have an interface to external media packages which are designed for process simulation. 


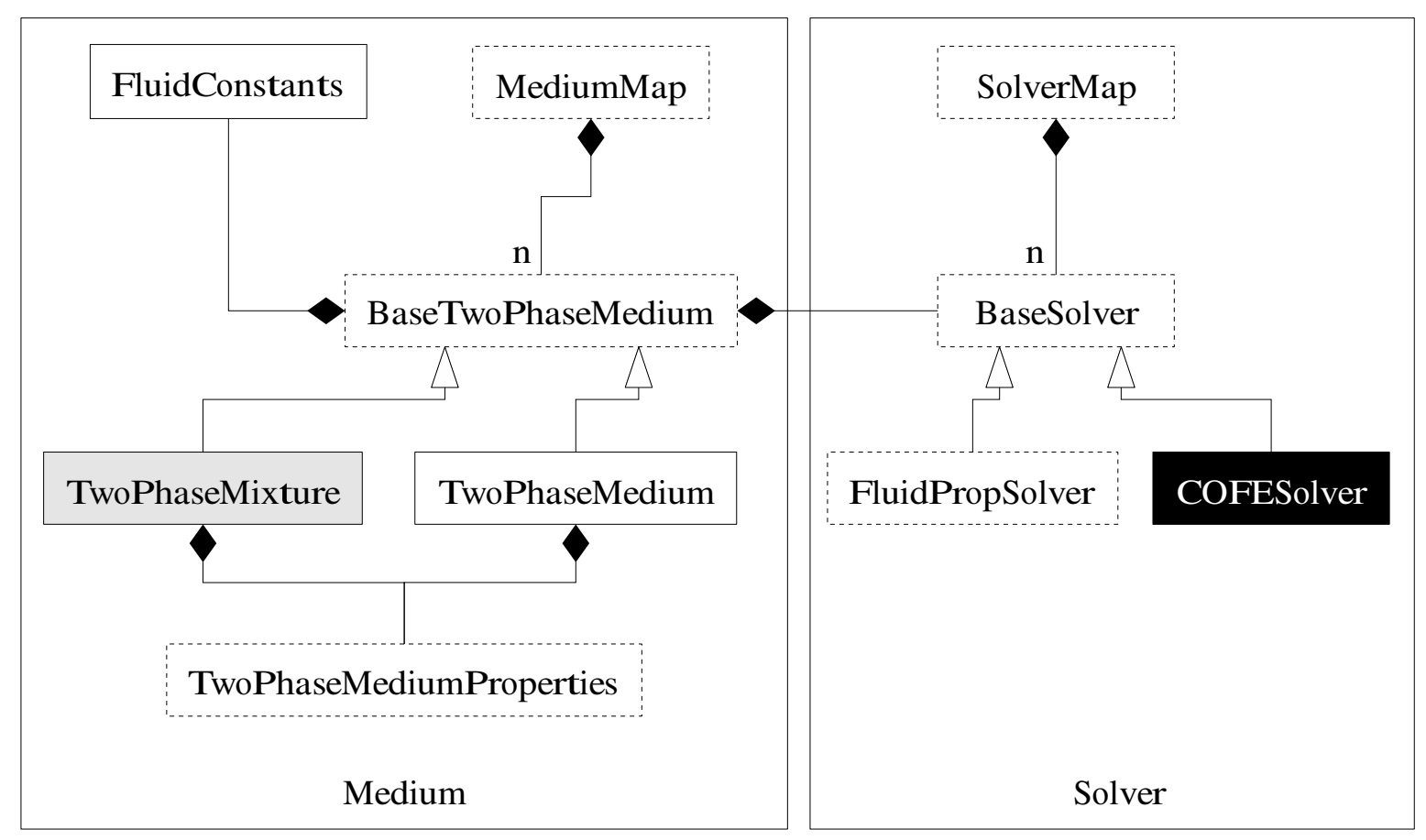

Figure 3: UML class diagram of the changed ExternalMedia C++ layer. A dotted box indicates a rewritten class. A new class is marked with a gray filled box or a black box, if it is in the planning stages.

For this purpose the interface standard CAPE-OPEN [1] has been developed by the CAPE-OPEN Laboratories Network at the beginning of this decade. [11] describe the use of such an interface to use thermodynamic and property data from an external tool in a Modelica model of a distillation column.

There are several commercial process engineering tools, which use this interface to exchange unit operation models or thermodynamic and physical property packages. One interesting tool is the free-of-charge COCO simulation environment [5], which contains TEA (COCO's Thermodynamics for Engineering Applications). TEA contains routines to calculate multiphase mixture properties. It also can access other property packages by the CAPE-OPEN interface. So there is the plan to implement a new solver object COFESolver to access TEA from the ExternalMedia library. This would allow Modelica models to access a wide range of simulation approved thermodynamic and physical property packages.

\subsection{Non-equilibrium Modeling}

One major problem using property packages of mixtures is that thermodynamic equilibrium is always assumed. That is for a certain composition $x_{\text {mix }}$ (see figure 4) the medium is decomposed in two phases with the equilibrium mole fractions $x_{\mathrm{A}}^{*}$ and $y_{\mathrm{A}}^{*}$. There- fore the thermodynamic properties like for instance the specific enthalpy for liquid and for vapor phase correspond the equilibrium compositions. However as in non-equilibrium models mass transfer is taken into account in one stage the medium is not decomposed in a liquid phase with $x_{\mathrm{A}}^{*}$ and a vapor phase $y_{\mathrm{A}}^{*}$ but in a liquid phase with $x_{\mathrm{A}}$ and a vapor phase with $y_{\mathrm{A}}$.

But most media models are not able to provide for instance the specific enthalpy at a temperature $\vartheta^{*}$ and a liquid composition $x_{\mathrm{A}}$, since in equilibrium the medium would not be single phase at these conditions.

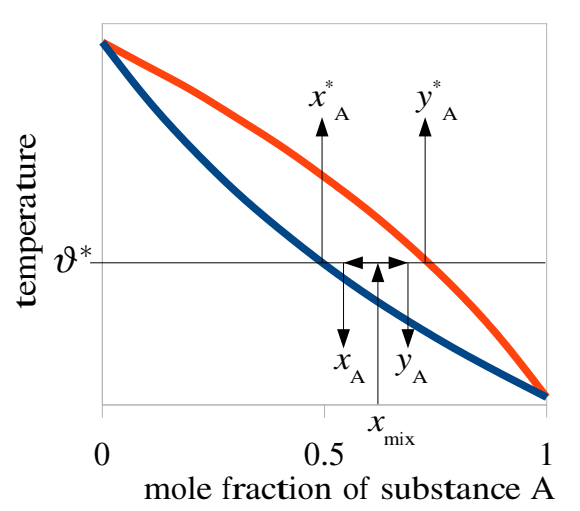

Figure 4: Decomposition of a mixture with mole fraction $x_{\text {mix }}$ into two phases with the composition $x_{\mathrm{A}}^{*}$ and $y_{\mathrm{A}}^{*}$ (mass and thermal equilibrium) or $x_{\mathrm{A}}$ and $y_{\mathrm{A}}$ (only thermal equilibrium between the two phases) 
For the current library the problem was solved in a way that the mixing of the different media is performed in the medium interface, so it is independent whether the properties are calculated in a Modelica model or external code. The mixing is performed ideal, where in case of a liquid phase for all components, whose bubble temperature is below the actual temperature, the enthalpy at boiling point is used. i.e. the enthalpy of liquid phase with a composition corresponding to a point in the two phase region $x_{\mathrm{A}}$ is computed the following way:

$$
h_{1}=x_{\mathrm{A}} \cdot h_{\mathrm{A}}^{\prime}+\left(1-x_{\mathrm{A}}\right) \cdot h_{\mathrm{B}}
$$

If the enthalpy of a vapor phase is computed, for all components, whose dew temperature is above the actual temperature, the enthalpy at dew point is used. So the vapor composition results in:

$$
h_{\mathrm{v}}=y_{\mathrm{A}} \cdot h_{\mathrm{A}}+\left(1-y_{\mathrm{A}}\right) \cdot h_{\mathrm{B}}^{\prime \prime}
$$

This introduces however an error in the thermophysical properties and a careful failure analysis has to be done before using the results.

\section{Example of Use}

In this section one example of use is presented. As an example the purification of flue gas of a waste incineration plant was chosen, since in this case media from ModelicaMedia can be used after some small modifications. The flue gas consists of a mixture of $\mathrm{N}_{2}, \mathrm{O}_{2}, \mathrm{H}_{2} \mathrm{O}, \mathrm{CO}_{2}, \mathrm{SO}_{2}, \mathrm{HF}$ and $\mathrm{HCl}$. In a first absorber this flue gas is brought in contact with water in order to primarily remove $\mathrm{HF}$ and $\mathrm{HCl}$. This absorber is modelled. The flue gas enters the absorber at the bottom, with a temperature well above $100{ }^{\circ} \mathrm{C}$; the water enters the column at the top, with a temperature around $50^{\circ} \mathrm{C}$. The absorber is a spray absorber, i.e. the necessary contact area is achieved by spraying small droplets of liquid in the air stream. The gases are then absorbed by the water, whereas water evaporates. At the column outlet a part of the liquid is purged, the rest is mixed with pure water and re-used. Such a system was for example also investigated by [8].

All gaseous components, beside $\mathrm{N}_{2}$ and $\mathrm{O}_{2}$ dissociate in the liquid phase. In this example, only the dissociation of $\mathrm{SO}_{2}$ is considered, since here the dissociation is strongest. The reaction equation is as follows:

$$
\mathrm{SO}_{2 \mathrm{aq}}+\mathrm{H}_{2} \mathrm{O} \Longleftrightarrow \mathrm{H}^{+}+\mathrm{HSO}_{3}^{-}
$$

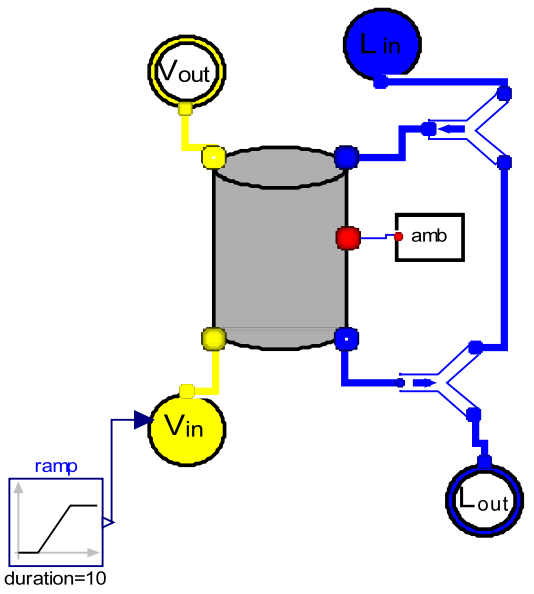

Figure 5: Modelling of a HF-HCl - absorber

For all gaseous components besides $\mathrm{H}_{2} \mathrm{O}$ equation (10) is used for the calculation of the phase equilibrium (where the Henry-coefficient was calculated temperature dependent), for water equation (9) is used. The components $\mathrm{H}^{+}$and $\mathrm{HSO}_{3}^{-}$do only exist in the vapour phase. Please note: not much emphasis was put on the task to gather all necessary parameters of all components (for example the necessary coefficients in order to calculate the temperature dependency of the Henry-constant). In this case reasonable assumptions were used. This example shall show in the first place that the simulation works and that it gives reasonable results.

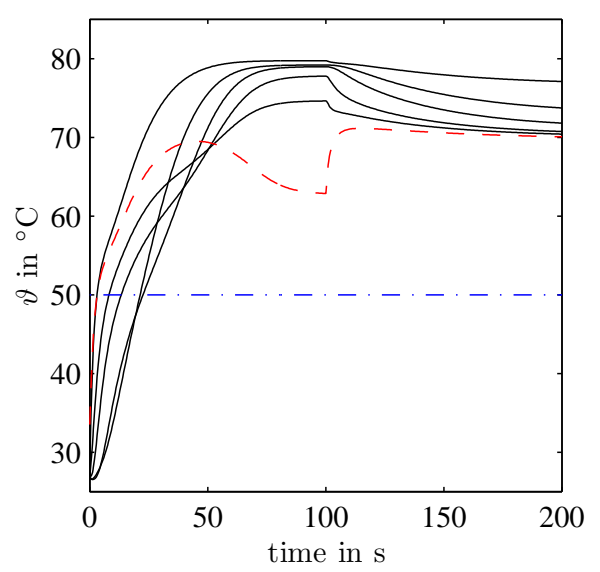

Figure 6: Vapour temperatures in the column (black lines) and temperature of fresh water (blue dot-anddashed line) and water at column inlet (red dashed line)

In this example the vapour flow inlet is halved at $t=100 \mathrm{~s}$ once steady state is obtained. Some results are shown in the following figures. Figure 6 shows the vapour temperature some of the stages. The highest 


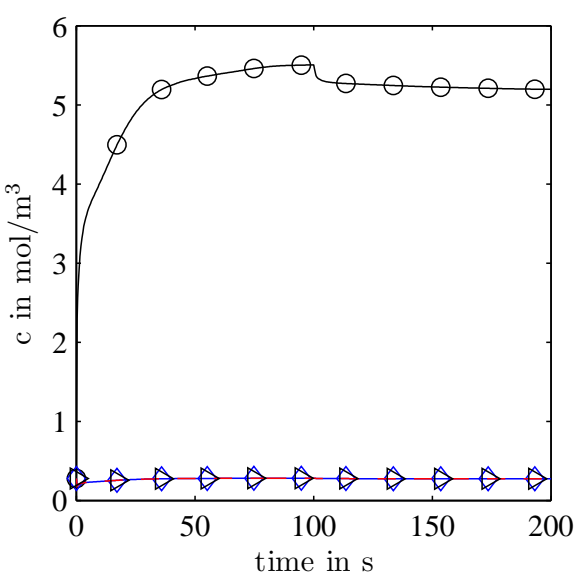

Figure 7: Liquid concentration of $\mathrm{SO}_{2}$ (black o), $\mathrm{H}^{+}$ (blue $\diamond)$ and $\mathrm{HSO}_{3}^{-}($red $\triangleright)$

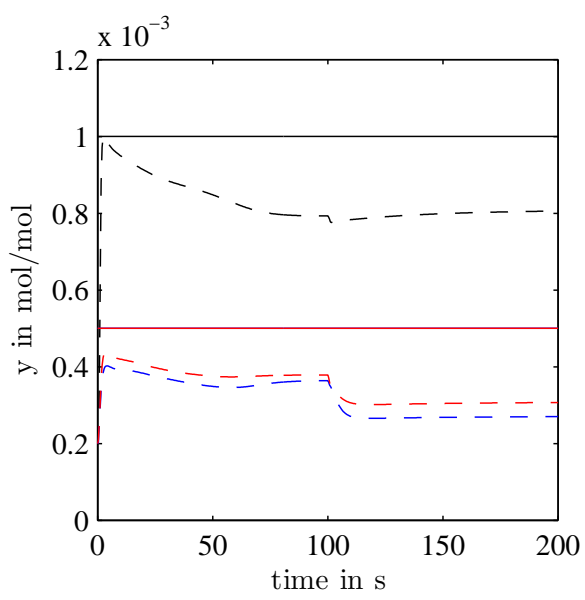

Figure 8: Vapour concentration of $\mathrm{SO}_{2}$ (black), $\mathrm{HCl}$ (blue) and HF (red) at column inlet (solid) and column outlet (dashed)

temperature correspond to stage 1 . The vapour enters the column at $144^{\circ} \mathrm{C}$, the fresh liquid water has a temperature of $50^{\circ} \mathrm{C}$ (blue dot-and-dashed line) the temperature of the recycled liquid water is denoted by the red dashed line (here, $80 \%$ of the water is recycled). Obviously the vapour temperature goes down when the vapour flow is reduced.

In figure 7 the concentration in the liquid of $\mathrm{SO}_{2}$, $\mathrm{H}^{+}$and $\mathrm{HSO}_{3}^{-}$is shown. As can be seen in the reaction equation, the concentration of $\mathrm{H}^{+}$and $\mathrm{HSO}_{3}^{-}$are equal. The ratio of $c_{\mathrm{H}^{+}} \cdot c_{\mathrm{HSO}_{3}^{-}}$to $c_{\mathrm{SO}_{2}}$ correspond to the equilibrium constant of the reaction.

Figure 8 finally shows the vapour concentration of the components to separate at the column inlet (solid line) as well as at the column outlet (dashed line). The concentration of $\mathrm{HCl}$ and $\mathrm{HF}$ at the column inlet are equal.

\section{Conclusion \& Outlook}

This paper proposed a Modelica-library for the modeling of simulation processes. The different column types can be used: spray, packed and tray column. All columns extend from the same base class where the mass and energy balance is established for the liquid as well as for the vapour phase. This also allows to calculate non-equilibrium states, where the thermodynamic equilibrium only exists at the phase boundary. The molar flow rate which results due to the difference between bulk concentration and the concentration at the phase boundary is calculated using empirical equations for mass transfer coefficients and interfacial area. However it is also possible to use an equilibrium model. In this case the Murphree tray efficiency can be used in order to describe the deviation from equilibrium in tray columns.

In order to avoid the implementation of medium models in Modelica, the ExternalMedia from [4] was adapted to account also for multi-phase multi-component mixtures. Since it turned out that the databases which can be easily accessed by the ExternalMedia are not suitable for process simulation, it is proposed to allow the use of the CAPOOPEN interface standard since this would give access to thermodynamic and physical property packages more suitable for process simulation.

\section{Nomenclature}

$A_{\text {free }}$

$a$

$c$
$\dot{L} \quad$ liquid volume flow rate in $\mathrm{m}^{3} / \mathrm{s}$ 


\begin{tabular}{|c|c|}
\hline$M$ & molar mass in $\mathrm{kg} / \mathrm{mol}$ \\
\hline$\dot{N}$ & molar flow rate in $\mathrm{mol} / \mathrm{s}$ \\
\hline$n$ & number of discrete elements \\
\hline$n S$ & number of substances \\
\hline$p$ & pressure in $\mathrm{Pa}$ \\
\hline$\dot{Q}$ & heat flow rate in $\mathrm{W}$ \\
\hline$T$ & temperature in $\mathrm{K}$ \\
\hline$t$ & time in $\mathrm{s}$ \\
\hline$u$ & specific inner energy in $\mathrm{mol} / \mathrm{r}$ \\
\hline$\dot{V}$ & vapour volume flow rate in $\mathrm{m}$ \\
\hline$V$ & volume of one element in $\mathrm{m}^{3}$ \\
\hline$v$ & velocity in $\mathrm{m} / \mathrm{s}$ \\
\hline$x$ & liquid composition in $\mathrm{mol} / \mathrm{mc}$ \\
\hline$y$ & vapour composition in $\mathrm{mol} / \mathrm{m}$ \\
\hline \multicolumn{2}{|c|}{ Greek symbols } \\
\hline$\varepsilon$ & hold up in $\mathrm{mol} / \mathrm{mol}$ \\
\hline$\varepsilon$ & void fraction \\
\hline \multicolumn{2}{|c|}{$\eta_{\text {Murphree }}$ tray efficieny of Murphree } \\
\hline$\gamma$ & activity coefficient \\
\hline$\phi$ & fugacity coefficient \\
\hline$\rho$ & density in $\mathrm{kg} / \mathrm{m}^{3}$ \\
\hline$\vartheta$ & temperature in ${ }^{\circ} \mathrm{C}$ \\
\hline$\zeta$ & drag factor \\
\hline
\end{tabular}

\section{Subscripts}

fromL coming from liquid phase

fromV coming from vapour phase

$\begin{array}{ll}i & \text { component } i \\ j & \text { stage } j \\ l & \text { liquid } \\ s & \text { solid } \\ t & \text { transfer } \\ v & \text { vapour }\end{array}$

\begin{tabular}{ll}
\multicolumn{2}{l}{ Superscripts } \\
bulk & bulk phase \\
$m$ & molar \\
sat & saturation \\
, & property at bubble point \\
$\prime \prime$ & property at dew point \\
$*$ & equilibrium
\end{tabular}

\section{References}

[1] The CAPE-OPEN Laboratories Network. http://www.colan.org/, visited on August 2009.

[2] Casella, Francesco and Alberto Leva: Modelica open library for power plant simulation: design and experimental validation. In Proceedings Modelica Conference 2003, pages 41-50, 2003.

[3] Casella, Francesco, Martin Otter, Katrin Prölß, Christoph Richter, and Hubertus Tummescheit: The Modelica Fluid and Media library for modeling of incompressible and compressible thermo-fluid pipe networks. In Proceedings Modelica Conference 2006, pages 631-640, 2006.

[4] Casella, Francesco and Christoph Richter: ExternalMedia: A Library for Easy Re-Use of External Fluid Property Code in Modelica. In Proceedings Modelica Conference 2008, pages 157161, 2008.

[5] COCO: cape open to cape open simulation environment. http://www. cocosimulator. org, visited on August 2009.

[6] FluidProp: Software for the calculation of thermophysical properties of fluids. http:// fluidprop.tudelft.nl/, visited on January 2009.

[7] Goedecke, Ralf (editor): FluidVerfahrenstechnik. Wiley-VCH, 2006.

[8] Malzkorn, Rainer: Simulation eines Rauchgassprühwäschwers mit aufwärts gerichteten Düsen. $\mathrm{PhD}$ thesis, Ruhr-Universität Bochum, 1999. 
[9] NIST Reference Fluid Thermodynamic and Transport Properties Database. http:// wWw.nist.gov/srd/nist23.htm, visited on June 2009.

[10] Reid and Prausnitz: The Properties of Gases \& Liquids. McGraw-Hill, 4th edition, 1987.

[11] Sandrock, Carl and Philip L. de Vaal: Dynamic simulation of Chemical Engineering systems using OpenModelica and CAPE-OPEN. In Jezowski, J. and J. Thullie (editors): 19th European Symposium on Computer Aided Process Engineering - ESCAPE19. Elsevier B.V./Ltd., 2009.

[12] Stichlmair, Johann: Grundlagen der Dimensionierung des Gas/Flüssigkeits-Kontaktapparates Bodenkolonne. Verlag Chemie, 1978.

[13] Swaaij, W.P.M. van and G.F. Versteeg: Mass Transfer Accompanied With Complex Reversible Chemical Reactions In Gas-Liquid Systems: An Overview. Chemical Engineering Science, 47:3181-3195, 1992. 